\title{
Limiting conditions for salt-fingering at an interface
}

\author{
Herbert E. HupperT* and Peter C. Manins*
}

(Received 9 June 1972; in revised form 30 October 1972; accepted 2 November 1972)

\begin{abstract}
Conditions for which salt fingers can be formed at the interface between hot, salty water above colder, fresher water are investigated both experimentally and theoretically. For experimental convenience, two solutes of different diffusivities, rather than heat and salt, were used. It is shown that an infinitesimal instability will occur and develop into a finite amplitude salt finger field provided that $R_{\rho}<\tau_{*} V \tau_{*}$. Here $R_{\rho}$ is the ratio of the increment in specific gravity due to the temperature difference across the interface to that due to the salinity difference and $\tau_{*}$ is the ratio of the diffusivity of heat to that of salt. For oceanic constituents $\tau_{*} \sqrt{ } \tau_{*} \sim 700$ while typical values of $R_{\rho}$ are very much less than this. There is thus sufficient potential energy stored in the salt field to form salt fingers in all parts of the ocean where hot, salty water overlies colder, fresher water.
\end{abstract}

\section{INTRODUCTION}

SALT FINGERING at the interface between a hot, salty layer and a lower cold, fresh layer of greater density has been subject to a number of experimental and theoretical studies. These studies have been partly stimulated by several oceanographic observations in which salt fingering is believed to play the major role in the creation and maintenance of the observed temperature and salinity structure. The measurements of TAIT and Howe $(1968,1971)$ and HowE and TAIT (1970) in the Northeast Atlantic, for example, indicated the existence of extensive series of statically stable layers of fairly uniform temperature and salinity at depths between 1200 and $1800 \mathrm{~m}$. These layers were separated by much thinner, horizontal interfaces, across which there were large temperature and salinity gradients in the sense required for the salt-finger mechanism to be operative. However, salt fingers have not actually been observed here, or elsewhere in the ocean, probably because appropriate instruments have not yet been developed. It seems fairly certain though, that salt fingers were present at the interfaces observed by Tait and Howe, and that they occur in many other oceanographic situations, not only those for which 'stepped' structures are the distinguishing feature.

Laboratory modelling of some of the observed structures using either heat and salt (TURNER, 1967) or two other stratifying components of different molecular diffusivities (STERN and TURNER, 1969, for example) has helped considerably in understanding some of the physical processes involved. In particular, the study of a single interface separating deep layers of hot salty water over cold fresh water has received much attention, both theoretical and experimental. TURNER (1973) discusses this work in greater detail.

For the purposes of this paper, the salt finger phenomenon may be illustrated by considering the following simple experiment. If a layer of hot, salty water is carefully poured on top of a layer of colder, fresher water of greater density, the fluid organises itself to form tall, thin cells, or fingers, at the interface. These fingers owe their existence to the capacity of descending elements to diffuse their heat horizontally more

*Department of Applied Mathematics and Theoretical Physics, University of Cambridge. 
rapidly than their salinity $\left[\kappa_{T}>\kappa_{S}\right.$, where $\kappa_{T}\left(\kappa_{S}\right)$ is the diffusivity of heat (salt) in water]. They thus become heavier than their surroundings and continue to descend. These motions occur even without the large diffusivity ratio, say $\tau_{*} \equiv \kappa_{T} / \kappa_{S}$, characteristic of a heat-salt system, for which $\tau_{*} \simeq 80$; similar motions can be seen using a layer of $\mathrm{NaCl}$ solution over a layer of $\mathrm{KCl}$ solution, for example, for which $\tau_{*} \simeq 1 \cdot 3$.

A full theoretical description of the motions within the interface of such an experiment has not yet been obtained. On the other hand, it is perhaps the ease with which fingers can be produced in the laboratory that has resulted in a failure to consider the circumstances under which fingers can not be formed. In this paper we address ourselves to this problem and ask: can a hot, salty layer above a cold, fresh layer be so hot that fingering will not occur? Or, alternatively, is it possible for the upper layer not to be sufficiently salty for fingering to exist? We report here a series of experiments designed to answer these questions, give a theoretical explanation of the results, and then discuss the oceanographic relevances of such limiting conditions for fingering at an interface.

Rather than using heat as one of the stratifying components with the concomitant problems of heat loss through the sides of the apparatus, we conducted our experiments using solutions of salt, cane sugar and Epsom-salts.* This permitted three different combinations of components to be used with values of $\tau_{*}$ approximately $1 \cdot 4$, $2 \cdot 1$ and $2 \cdot 9$. Labelling the solution of larger (smaller) diffusivity as the $T(S)$ solution, we performed a series of experiments in which an $S$ solution was poured over a more dense $T$ solution. The experiments showed that fingering could not exist for sufficiently large values of the stability ratio $R_{\rho} \equiv \alpha \Delta T / \beta \Delta S$, where $\alpha \Delta T(\beta \Delta S)$ is the increment of the original specific gravity of the $T(S)$ solution over that of fresh water. From the results, presented in Fig. 1, the limit of fingering is seen to be consistent with $R_{\rho}=\tau_{*} \sqrt{ } \tau_{*}$.

After presenting the details of the experiments in the next section, we explain the results by calculating the effects of diffusion on an originally sharp interface. Diffusion of both components produces a $T$ and $S$ distribution to which we apply previously obtained results on infinitesimal instability. We next show, using a simple model for finite amplitude fingering, that the conditions which allow infinitesimal disturbances to grow will also permit finite amplitude motions.

\section{THE EXPERIMENTS}

A total of 42 experiments was performed in two identical, rectangular, perspex containers of internal dimensions $5 \cdot 0 \times 15 \cdot 2 \times 12.0 \mathrm{~cm}$ high. To preclude the occurrence of thermal effects all $T$ and $S$ solutions used were prepared at room temperature $\left(\mathrm{ca} .20^{\circ} \mathrm{C}\right.$ ) and were within $1 \mathrm{C}^{\circ}$ of each other. In order to determine the specific gravity of each solution, the refractive index was measured by a temperature compensating hand refractometer $\dagger$ and this reading converted to specific gravity using the tables in WEAST (1971), by fitting a linear least squares interpolation through the tabulated data in the vicinity of the measured refractive index. For each experiment

*Some exploratory experiments were also performed involving solutions of $\mathrm{HCl}$ and $\mathrm{KCl}$. However, the large heats of dilution, positive for $\mathrm{HCl}$ and negative for $\mathrm{KCl}$, introduced an unwanted third component, heat. These experiments were therefore not considered further.

†American Optical Co. T/C Refractometer Type 10419. 
$400 \mathrm{~cm}^{3}$ of the $T$ solution were poured into one of the containers and a $\frac{1}{2} \mathrm{~cm}$ thick foam strip, presoaked in the $S$ solution, was gently placed on to the surface of the $T$ solution. Then $400 \mathrm{~cm}^{3}$ of the $S$ solution were carefully fed evenly over the surface of the float. Upon completion of this filling process, requiring some 10 minutes, the float was left in position to act as a lid.

Visualisation of the filling process and subsequent motion was by means of a shadowgraph, relying on the different refractive indices of the two solutions. The interface region, defined as the region of large refractive index - and hence densitygradients, was observed to be practically undisturbed by the method of filling, and by the time the filling process was completed was generally less than $2 \mathrm{~mm}$ thick. This thickness was seen gradually to increase with time owing to the effects of diffusion. In a number of experiments this is all that was seen. In the remainder, the presence of fingers was also visible over at least half of the interface length. A majority of the interfaces which produced fingers did so within 10 minutes of completion of filling, though all experiments were continued for at least 30 minutes. If there was any doubt whether fingers were present or not, this was extended to 60 minutes, although no experiment changed its character in this time.

The results of the separate experiments with salt and sugar, Epsom-salts and sugar, and salt and Epsom-salts are shown in Fig. 1. The symbol $\bullet$ is used to signify fingering along the whole interface length, $O$ to signify fingering, generally in clumps, along most of the interface, and $x$ to signify no evident motion other than the diffusive spreading of the interface. We interpret only the points marked with an $x$ as indicating that fingering is impossible under the stated conditions. Also marked in Fig. 1 is the value at which $R_{\rho}$ equals $\tau_{*} \sqrt{ } \tau_{*}$, where the several diffusivities were estimated as follows. The diffusivity of a solute was assumed independent of the presence of the other solute although this is known not to be strictly true.* For the sugar and salt solutions, the diffusivities were taken from Gosting and Morris (1949) and Stokes (1950), respectively, for a solution concentration equal to a half that originally measured - that is, equal to the mean value expected in the interface region. For the Epsomsalts solutions, no tabulation of its diffusivity as a function of concentration in the appropriate range could be found (diffusivities at concentrations of approximately $0.09 \mathrm{~mole} / \mathrm{l}$. were required), so the value determined by HARNED and HuDSON (1951) at the highest concentration they investigated $(0.006$ mole $/ 1$.) was used. Finally, all the values, tabulated at $25^{\circ} \mathrm{C}$, were corrected for use at $20^{\circ} \mathrm{C}$ by applying the formula

$$
\kappa_{20}=0.874 \kappa_{25},
$$

where $\kappa_{20}\left(\kappa_{25}\right)$ signifies the diffusivity at $20^{\circ} \mathrm{C}\left(25^{\circ} \mathrm{C}\right)$. This procedure led to the approximate values of $1.3 \times 10^{-5}, 0.45 \times 10^{-5}$ and $0.61 \times 10^{-5} \mathrm{~cm}^{2} \mathrm{sec}^{-1}$ for the diffusivities of the salt, sugar and Epsom-salts solutions, respectively. They are thought to be correct to within $\pm 5 \%$ for the actual conditions of the experiments. This gives an uncertainty for $\tau_{*} \sqrt{ } \tau_{*}$ of $\pm 15 \%$.

\footnotetext{
*See Reinfelds and Gosting (1964) for some details of the manner in which sugar and $\mathrm{KCl}$ interact in aqueous solution, and STOKES and STOKES (1956) for the modification to the diffusion of electrolytes including $\mathrm{NaCl}$ in sucrose solutions. Unfortunately, these studies are not directly applicable to the systems studied in the present paper.

$\uparrow$ Tpecifically, $\kappa_{20}=\left(A_{20} / A_{25}\right)\left(\eta_{25} / \eta_{20}\right) \kappa_{25}$, the Stokes-Einstein equation (see, for example, Gosting and Morris, 1949), where $A$ is the temperature in degrees absolute, $\eta$ the dynamic viscosity of water, and the subscript indicates the relevant temperature in degrees $\mathbf{C}$.
} 

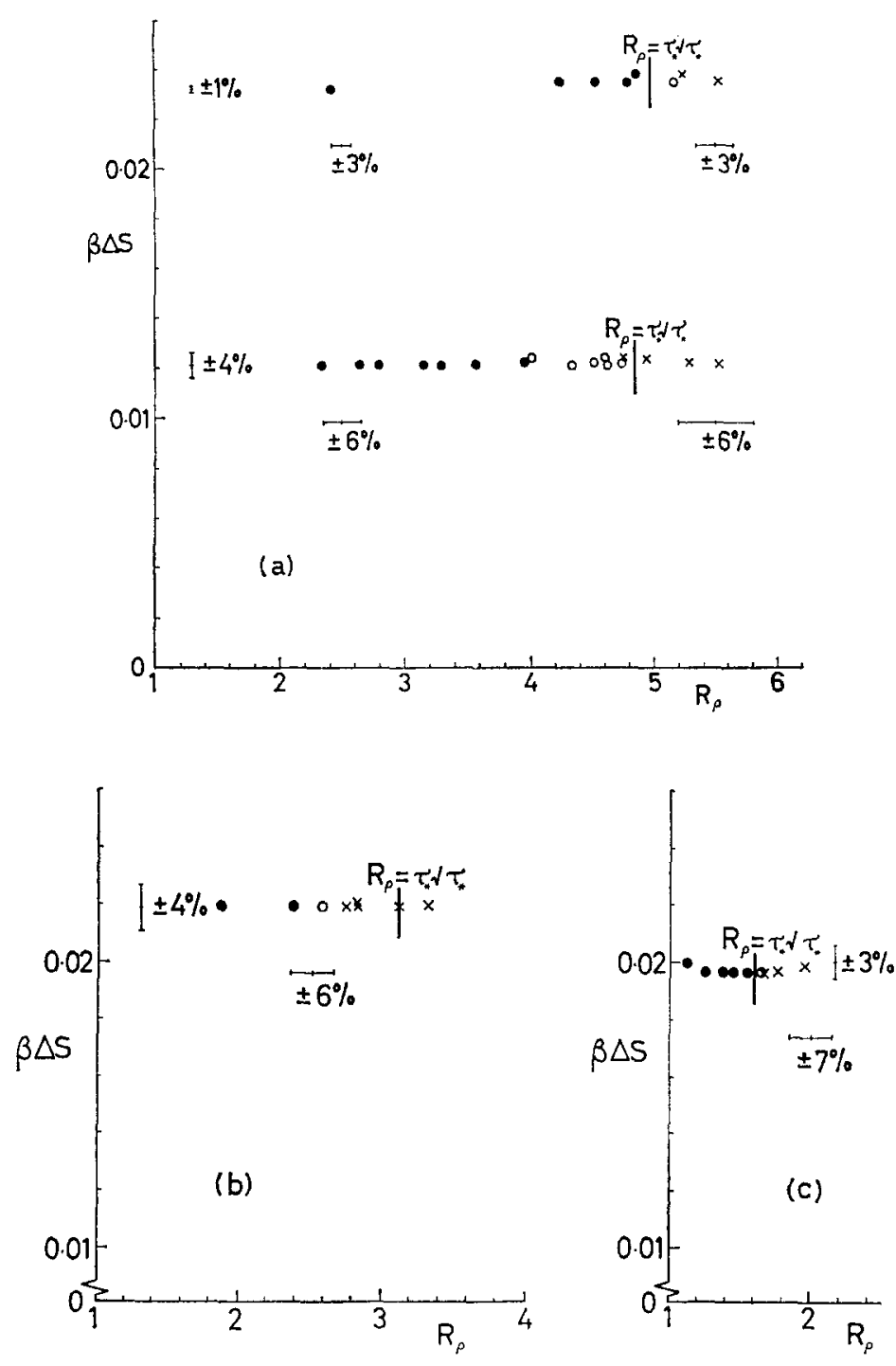

Fig. 1. The results of the experiments with solutions of : (a) sugar over salt $\left(\tau_{*} \simeq 2 \cdot 9\right)$; (b) Epsom-salts over salt $\left(\tau_{*} \simeq 2 \cdot 1\right)$; and (c) sugar over Epsom-salts $\left(\tau_{*} \simeq 1 \cdot 4\right)$. The symbol signifies that fingers were seen along the whole interface, $\bigcirc$ signifies that fingers were seen along most of the interface and $x$ signifies no fingers were seen. The vertical error bars refer to measurements of $\beta \Delta S$ and the horizontal error bars to measurements of $R_{\rho}=\alpha \Delta T / \beta \Delta S$.

It appears from the separate results that $\tau_{*} \sqrt{ } \tau_{*}$ represents a good measure for the limit of fingering: for $R_{\rho}>\tau_{*} \sqrt{ } \tau_{*}$ fingering cannot occur at an interface. This result appears not to be as accurate for the salt, Epsom-salts experiment, for which it is thought that the use of two electrolytes and the accompanying presence of free ions on both sides of the interface played a part in the process of diffusion. A theoretical explanation for the value $\tau_{*} \sqrt{ } \tau_{*}$ is presented in the next section. 
THEORETICAL EXPLANATION

\section{Infinitesimal analysis}

The instability of a fluid in which there are two components of different molecular diffusivities with opposing, linear concentration gradients has been studied by, amongst others, STERN (1960) and BAINES and GILL (1969). Part of their analysis concentrated on the situation for which, with $z$ directed vertically upwards, $-\mathrm{d} T / \mathrm{d} z$ and $\mathrm{d} S / \mathrm{d} z$ were both positive and constant. They proved then that instability to an infinitesimal disturbance between two stress-free, horizontal planes of separation $h$, conducting to both $T$ and $S$ can occur only if

$$
\tau_{*} R_{S}>R_{T}+\frac{27}{4} \pi^{4}
$$

where

$$
\begin{aligned}
& R_{T}=-\alpha g h^{4} \frac{\mathrm{d} T}{\mathrm{~d} z} /\left(\kappa_{\mathrm{T}} v\right)>0, \\
& R_{S}=\beta g h^{4} \frac{\mathrm{d} S}{\mathrm{~d} z} /\left(\kappa_{T} v\right)>0,
\end{aligned}
$$

$g$ is the gravitational acceleration and $v$ the kinematic viscosity of the fluid. If both $R_{T}$ and $\tau_{*} R_{S}$ are very much larger than $27 \pi^{4} / 4$, the second term on the right-hand side of (2) may be neglected and, using (3) and (4), the condition for instability can be written as

$$
G \equiv-\alpha \frac{\mathrm{d} T}{\mathrm{~d} z} / \beta \frac{\mathrm{d} S}{\mathrm{~d} z}<\tau_{*}
$$

This analysis for infinitesimal instability may be applied to interpret our experiments after the diffusive spreading of the interface has been considered. Placing the origin of co-ordinates at the initial position of the interface, we can write the initial $T$ and $S$ distributions as

$$
\begin{aligned}
\{T, S\} & =\{\Delta T, 0\} & & (-H<z<0) \\
& =\{0, \Delta S\} & & (0<z<H), *
\end{aligned}
$$

where $H$ represents the depth of each layer. After a time $t$, the $T$ and $S$ distributions are

$$
\{T, S\}=\left\{\frac{1}{2} \Delta T \operatorname{erfc}\left[\frac{1}{2} z /\left(\kappa_{T} t\right)^{1 / 2}\right], \frac{1}{2} \Delta S \operatorname{erfc}\left[-\frac{1}{2} z /\left(\kappa_{S} t\right)^{1 / 2}\right]\right\},
$$

where we have set $H=\infty$, concentrating on values of $z$ and $t$ sufficiently small that the influence of the horizontal boundaries can be neglected. Differentiating (7), we find that

$$
G=\tau_{*}^{-1 / 2} R_{p} \exp \left[\frac{1}{4} z^{2} t^{-1}\left(\kappa_{S}^{-1}-\kappa_{T}^{-1}\right)\right],
$$

which has a minimum value at $z=0$ equal to $\tau_{*}{ }^{-1 / 2} R_{\rho}$. We now use this value in (5b) to determine the maximum value of $R_{\rho}$ for which instability can occur. We do this even though the conditions for which (5b) has been derived are not exactly those of our

*The analysis can be generalised to allow for an initial interfacial structure of thickness $\delta$, say. The final results are unchanged, however, if the diffusive spreading takes place for a time much larger than $A \delta^{2}\left(\kappa_{S}^{-1}-\kappa_{T}^{-1}\right)$, where $A$ is a constant dependent on the initial structure. Our experiments were run for a time sufficient to satisfy this requirement for values of $A$ corresponding to reasonable initial conditions. 
experiments, for which a direct analysis would be far more difficult than the present context warrants. However, we anticipate that $(5 \mathrm{~b})$ would not be very much altered by such analysis. With this procedure we conclude that instability can occur only if

$$
R_{\rho}<\tau_{*} \sqrt{ } \tau_{*} .
$$

Two of the assumptions involved in the determination of (9)-the setting of $H$ equal to infinity and the neglect of $27 \pi^{4} / 4$ with respect to $R_{T}$ and $\tau_{*} R_{S}$-can be justified for our experiments. The former assumption requires the interface thickness to be very much less than $H$ and the time $t$ to be very much less than $H^{2} / \kappa_{T}$. Both of these requirements were easily satisfied. To evaluate $R_{T}$ and $\tau_{*} R_{S}$ we substitute into (4) and (5) the representative values (in cgs units) $g \sim 10^{3},-\alpha \mathrm{d} T / \mathrm{d} z \sim \beta \mathrm{d} S / \mathrm{d} z \sim 10^{-1}$, $\kappa_{T} \sim \kappa_{S} \sim 10^{-5}, v \sim 10^{-2}$ and $h \sim 0.2$ (an underestimate of the interfacial thickness when fingering occurred) to obtain $R_{T} \sim \tau_{*} R_{S} \sim 10^{6}$, which is much larger than $27 \pi^{4} / 4$.

\section{Finite amplitude analysis}

Condition (9) has been determined by using (5b), valid for infinitesimal disturbances. It will be shown below that the simplest conceivable mode for finite amplitude fingers leads to a condition analogous to (5b), viz.

$$
-\alpha \frac{\mathrm{d} \bar{T}}{\mathrm{~d} z} / \beta \frac{\mathrm{d} \bar{S}}{\mathrm{~d} z}<\tau_{*}
$$

where an overbar represents a horizontal average. Equations (5b) and (10) taken together suggest that (9) not only permits an infinitesimal instability to exist but also permits subsequent finite amplitude motion to persist (for some time, at least).

The major assumptions involved in our model are that we take the fingers to be steady and the velocity to be entirely vertical. Continuity then requires the vertical velocity, say $w$, to be a function of $x$ and $y$ only and the horizontal momentum equations require the pressure to be a function of $z$ only. With the Boussinesq approximation the vertical momentum equation then becomes

where the density

$$
\frac{1}{\rho_{0}} \frac{\mathrm{d} p}{\mathrm{~d} z}=-g(1+\alpha T+\beta S)+v \nabla_{2}{ }^{2} w(x, y),
$$

and

$$
\rho=\rho_{0}(1+\alpha T+\beta S)
$$

$$
\nabla_{2}^{2} \equiv \frac{\partial^{2}}{\partial x^{2}}+\frac{\partial^{2}}{\partial y^{2}}
$$

It hence follows that $\alpha T+\beta S$ must be of the form

$$
\alpha T+\beta S=f(z)+h(x, y),
$$

for some $f(z)$ and $h(x, y)$. We now extend (14) and assume that $\alpha T$ and $\beta S$ can be expressed individually as functions only of $z$ plus functions only of $x$ and $y$ : in particular,

and

$$
\alpha T=f_{T}(z)+h_{T}(x, y)
$$

$$
\beta S=f_{S}(z)+h_{S}(x, y),
$$


where $h_{T}$ and $h_{S}$ are defined such that $\bar{h}_{T}=\bar{h}_{S}=0$. Introducing (15) and (16) into the equations for the conservation of $T$ and $S$, we obtain

$$
w(x, y) \frac{\mathrm{d} f_{T}}{\mathrm{~d} z}=\kappa_{T}\left[\frac{\mathrm{d}^{2} f_{T}}{\mathrm{~d} z^{2}}+\nabla_{2}^{2} h_{T}(x, y)\right]
$$

and

$$
w(x, y) \frac{\mathrm{d} f_{S}}{\mathrm{~d} z}=\kappa_{S}\left[\frac{\mathrm{d}^{2} f_{S}}{\mathrm{~d} z^{2}}+\nabla_{2}^{2} h_{S}(x, y)\right] .
$$

Since continuity requires $\bar{w}=0$, taking the horizontal average of (17) and (18) and integrating shows that

$$
\frac{\mathrm{d} f_{T}}{\mathrm{~d} z}=\gamma_{T} \quad \text { and } \quad \frac{\mathrm{d} f_{S}}{\mathrm{~d} z}=\gamma_{S}
$$

where $\gamma_{T}$ and $\gamma_{S}$ are constants. Subtracting (11) from its horizontal average and substituting (15) and (16) into the result, we obtain

$$
0=g\left[h_{T}(x, y)+h_{S}(x, y)\right]-v \nabla_{2}^{2} w
$$

while substitution of (19) into (17) and (18) yields

and

$$
\gamma_{T} w=\kappa_{T} \nabla_{2}^{2} h_{T}(x, y)
$$

$$
\gamma_{S} w=\kappa_{S} \nabla_{2}^{2} h_{S}(x, y)
$$

The system (20) to (22) is now linear and has solutions of the form

$$
\left[w(x, y), h_{T}(x, y), h_{S}(x, y)\right]=\left[W, H_{T}, H_{S}\right] \cos m x \cos m y .
$$

Substituting (23) into (20) to (22), we find that

$$
\left(m^{2}+n^{2}\right)^{2}=\frac{g}{v}\left(\frac{\gamma_{T}}{\kappa_{T}}+\frac{\gamma_{S}}{\kappa_{S}}\right)
$$

For a solution to be possible the right-hand side of (24) must be positive, that is (with $\gamma_{T}$ negative)

$$
-\gamma_{T} / \gamma_{S}<\tau_{*}
$$

which in terms of the original $T$ and $S$ fields can be written as

$$
-\alpha \frac{\mathrm{d} \bar{T}}{\mathrm{~d} z} / \beta \frac{\mathrm{d} \bar{S}}{\mathrm{~d} z}<\tau_{*}
$$

although with the representation (15) and (16) for the $T$ and $S$ fields the overbars in (26) may be omitted.

Conditions (5b) and (26), although so similar in appearance, are not derivable one from the other. The former is valid for an infinitesimal disturbance with a specific, $z$-dependent vertical velocity and predicts the conditions for which the onset of instability is possible. The latter arises from attempting to model finite amplitude fingers well away from either of their ends and determines an upper limit on the ratio of the vertical gradients within the finger field. And this same upper limit holds for the initial onset of instability. This suggests that as long as the ends of the fingers have no 
significant effect on their existence, an instability once established will persist to finite amplitude, although the $T$ and $S$ fields may be thereby modified.

Condition (26) can be written in an alternative form incorporating the mean $T$ and $S$ buoyancy fluxes

and

$$
F_{T}=\rho_{0} \alpha \overline{w T}=\frac{1}{2} \rho_{0} W H_{T}
$$

and the flux ratio $†$

$$
F_{S}=\rho_{0} \beta \overline{w S}=\frac{1}{2} \rho_{0} W H_{S}
$$

$$
R_{F} \equiv-F_{T} / F_{S}=-H_{T} / H_{S}
$$

Dividing (21) by (22) after substituting (23) into each, we obtain

$$
R_{F}=-\gamma_{T} /\left(\tau_{*} \gamma_{S}\right)
$$

Then upon substitution of (25) into (30), the condition for the existence of finite amplitude fingers becomes

$$
R_{F}<1 \text {. }
$$

Since $R_{F}$ represents the ratio of the potential energy gained by the $\mathrm{T}$ field to that released by the $S$ field, it must be less than unity, the deviation from unity being the proportion of released energy that is dissipated by viscosity. Equation (31) thus states that the limiting condition for the formation of salt fingers at an interface occurs when all the potential energy released by one component is taken up by the other.

\section{CONCLUSIONS}

In the ocean, where heat and salt are the two components, $\tau_{*} \simeq 80$ and hence $\tau_{*} \sqrt{ } \tau_{*} \simeq 700$. Extrapolating our experimental and theoretical results on the basis that both the thermal and saline Rayleigh numbers are much larger than $27 \pi^{4} / 4$, we conclude that stability ratios less than 700 will lead to salt fingering. Taking $\alpha \sim 10^{-4} / \mathrm{C}^{\circ}$ and $\beta \sim 7 \times 10^{4} / \%$, we hence calculate that salt fingering at an interface is possible when the salinity difference exceeds $2 \times 10^{-4} \%$ per $\mathrm{C}^{\circ}$ temperature difference.

Oceanic values of $R_{\rho}$ are around 1; for Tait and Howe's measurements, for example, $R_{\rho} \simeq 1 \cdot 2$. Thus the condition $R_{\rho}<\tau_{*} \tau_{*} \simeq 700$ should be satisfied in all parts of the ocean where hot, salty water overlies colder, fresher water.

Acknowledgements-This work has benefited from a number of discussions with Dr. J. S. TURNER and Dr. P. F. LINDEN. It was supported in part by a grant from the British Admiralty. The second author acknowledges the support of a Commonwealth Scholarship and aid from an Australian Public Service Financial Assistance.

\section{REFERENCES}

Baines P. G. and A. E. Gill (1969) On thermohaline convection with linear gradients. J. Fluid Mech., 37, 289-306.

Gosting L. J. and M. S. MoRRIS (1949) Diffusion studies on dilute aqueous sucrose solution at 1 and $25^{\circ}$ with the Gouy interference method. $J$. Am. chem. Soc., 71, 1998-2006.

HARNED H. S. and R. M. HudSON (1951) The diffusion coefficient of magnesium sulphate in dilute aqueous solutions at $25^{\circ}$. J. Am. chem. Soc., 73, 5880-5882.

$\dagger$ As defined $R_{F}$ is positive because the flux of the $T$ component is in the opposite direction to that of the $S$ component. 
HowE M. R. and R. I. TAIT (1970) Further observations of thermohaline stratification in the deep ocean. Deep-Sea Res., 17, 963-972.

REINFELDS G. and L. J. Gosting (1964) Measurements of isothermal diffusion at $25^{\circ} \mathrm{C}$ with the Gouy diffusiometer on the system water-sucrose-KCl. J. phys. Chem., 68, 2464-2470.

Stern M. E. (1960) The 'salt-fountain' and thermohaline convection. Tellus, 12, 172-175.

STERN M. E. and J. S. TURNER (1969) Salt fingers and convecting layers. Deep-Sea Res., 16, 497-511.

Stokes Jean M. and R. H. Stokes (1956) The conductances of some simple electrolytes in aqueous sucrose solutions at $25^{\circ}$. J. phys. Chem., 60, 217-220.

Stokes R. H. (1950) The diffusion coefficients of eight univalent electrolytes in aqueous solution at $25^{\circ}$ C. J. Am. chem. Soc., 72, 2243-2247.

TAIT R. I. and M. R. Howe (1968) Some observations of thermohaline stratification in the deep ocean. Deep-Sea Res., 15, 275-280.

TAIT R. I. and M. R. Howe (1971) Thermohaline staircase. Nature, Lond., 231, 178-179.

Turner J. S. (1967) Salt fingers across a density interface. Deep-Sea Res., 14, 599-611.

TURner J. S. (1973) Buoyancy effects in fluids. Cambridge University Press, $367 \mathrm{pp}$.

WEAST R. C., editor (1971) Handbook of chemistry and physics. 52nd edition. The Chemical Rubber Co., $1263 \mathrm{pp}$. 